\title{
Corrigendum: Ultra-High Field NMR and MRI-The Role of Magnet Technology to Increase Sensitivity and Specificity
}

\author{
Ewald Moser ${ }^{1,2 *}$, Elmar Laistler ${ }^{1,2}$, Franz Schmitt $^{3}$ and Georg Kontaxis ${ }^{4}$ \\ ${ }^{1}$ Center for Medical Physics and Biomedical Engineering, Medical University of Vienna, Vienna, Austria, ${ }^{2}$ High-Field MR \\ Center, Medical University of Vienna, Vienna, Austria, ${ }^{3}$ Lakeside Imaging/e, Plau am See, Germany, ${ }^{4}$ Max F. Perutz \\ Laboratories, Department of Structural and Computational Biology, Center for Molecular Biology, University of Vienna, \\ Vienna, Austria
}

Keywords: NMR, MRI, MRS, magnet technology, superconductors, gradients, sensitivity

\section{A corrigendum on}

Ultra-High Field NMR and MRI-The Role of Magnet Technology to Increase Sensitivity and Specificity

by Moser, E., Laistler, E., Schmitt, F., and Kontaxis, G. (2017). Front. Phys. 5:33. doi: 10.3389/fphy.2017.00033

OPEN ACCESS

Edited by:

Evren Özarslan,

Linköping University, Sweden

Reviewed by:

Alexander Rauscher,

University of British Columbia, Canada

*Correspondence:

Ewald Moser

ewald.moser@meduniwien.ac.at

Specialty section:

This article was submitted to

Biomedical Physics,

a section of the journal

Frontiers in Physics

Received: 26 August 2017

Accepted: 31 August 2017

Published: 12 September 2017

Citation:

Moser E, Laistler E, Schmitt F and Kontaxis G (2017) Corrigendum: Ultra-High Field NMR and MRI-The

Role of Magnet Technology to Increase Sensitivity and Specificity.

Front. Phys. 5:41.

doi: 10.3389/fphy.2017.00041
In the original article, the references [10] and [69] are identical. It should appear only once.

In the original article, there was an error (Prof. Lauterbur's idea was never patented but certified).

A correction has been made to RESISTIVE ELECTROMAGNETS, THE BEGINNING OF ANALYTICAL NMR, Paragraph Number 10:

On 2 September 1971, Paul C. Lauterbur, a professor of chemistry at the State University of New York at Stony Brook, had his idea of applying magnetic field gradients in all three dimensions to create NMR images certified.

In the original article, there was an error (interections).

A correction has been made to PERMANENT MAGNETS, THE LOW COST ALTERNATIVE, Paragraph Number 2:

Erik Odeblad, a gynecologist, after his stay at Stanford at about the time of Bloch's discovery, went back to Stockholm and, together with G. Lindstroem, built his own low-resolution NMR spectrometer. Widely unnoticed by the scientific NMR community they found out, and already published in 1955 [27], that different tissues had distinct relaxation times, most likely due to different water content but also due to different compartmentalization and interactions with lipids - a phenomenon that explains tissue contrast in MR imaging. Odeblad continued working on human fluids and tissues throughout the following decades and a large number of scientific papers on NMR relaxation in human tissues and secretions of mucous membranes followed until 1968 [22].

In the original article, there was an error (FDA approval, instead of correctly CE-labeling).

A correction has been made to SUPERCONDUCTING ELECTROMAGNETS, THE GOLDEN AGE OF NMR, Clinical MRI and MR Research Using Whole-Body Superconductors, Paragraph Number 5:

Supporting particularly the booming field of functional MRI including BOLD-based contrast, started in the early 1990s, 3 T systems with strong and fast gradient coils have been developed in parallel and the first "high end" routine MRI systems operating at that field strength became 
commercially available in 2000. Together with "parallel imaging," i.e., array coils and SENSE [64]/GRAPPA [65] techniques, this move turned out to be extremely successful and quickly, i.e., within 10 years, $20 \%$ of all MRI sales are $3 \mathrm{~T}$. BOLD-based fmri, greatly benefitting from the increased $\mathrm{B} 0$ and faster/stronger gradients, was the driving force for developments and sales of $3 \mathrm{~T}$ systems. The following decade in clinical routine and research was characterized by a plateau in terms of newly installed systems (about 3,000 units p.a.), where $3 \mathrm{~T}$ systems started replacing the lower field units. Robust and strong/fast gradient coils and efficient phased-array RF-coils (requiring multi-channel receive electronics) helped to constantly improve (fast) imaging performance. Again, the higher sensitivity achievable could be used for speed and increasing specificity, now also via multiparametric imaging, e.g., combining structural and functional MRI, resulting in exam times still well tolerated by patients. For high resolution, localized MR spectroscopy of non-proton nuclei (e.g., ${ }^{31} \mathrm{P},{ }^{13} \mathrm{C}$ ), the sensitivity was still too low to incorporate such a protocol into clinical routine. By 2015, about $60 \%$ of all clinical MRI volume sales were achieved for $1.5 \mathrm{~T}, 34 \%$ for $3 \mathrm{~T}$ systems (slowly replacing current $1.5 \mathrm{~T}$ scanners), and a rather stable $6 \%$ for low field scanners $(<0.5 \mathrm{~T})$. Although a few 4 $\mathrm{T}$ and $4.7 \mathrm{~T}$ prototype magnets have been installed since 1988, stronger magnets have been operating at $7 \mathrm{~T}$ for UHF research only $[57,58,61,66,67]$. Thus, $7 \mathrm{~T}$ human whole body scanners are currently only being installed in selected high end (clinical) research units (with about 70 systems installed so far). Currently, CE-labeling but not yet FDA approval has been obtained only for Siemens' $7 \mathrm{~T}$ Terra system, centered around an active shielded compact magnet and standard operating software/ console.

The authors apologize for these errors and state that this does not change the scientific conclusions of the article in any way.

Conflict of Interest Statement: The authors declare that the research was conducted in the absence of any commercial or financial relationships that could be construed as a potential conflict of interest.

Copyright (c) 2017 Moser, Laistler, Schmitt and Kontaxis. This is an open-access article distributed under the terms of the Creative Commons Attribution License (CC $B Y)$. The use, distribution or reproduction in other forums is permitted, provided the original author(s) or licensor are credited and that the original publication in this journal is cited, in accordance with accepted academic practice. No use, distribution or reproduction is permitted which does not comply with these terms. 